\title{
Title: Listening to Long COVID: Epistemic Injustice and COVID-19 morbidity
}

Vivian V. Altiery De Jesús $(\mathrm{MBE})^{1}$, Nisreen Alwan $(\mathrm{PhD})^{23}{ }^{4}$, Felicity Callard(PhD $)^{5}$, Zackary Berger ( M.D.) $)^{6,7}$

1. University of Puerto Rico School of Medicine

2. School of Primary Care, Population Sciences and Medical Education, Faculty of Medicine, University of Southampton, Southampton, UK

3. NIHR Southampton Biomedical Research Centre, University of Southampton and University Hospital Southampton NHS Foundation Trust, Southampton, UK

4. NIHR Applied Research Collaboration (ARC) Wssex, UK

5. University of Glasgow

6. Johns Hopkins Berman Institute of Bioethics

7. Johns Hopkins School of Medicine

\section{Corresponding Author}

Zackary Berger

Email: zberger1@jhmi.edu

Phone: 410-955-5000

\section{Word Count}

2,094 (Article); 178 (Abstract)

\section{Keywords:}

Long COVID, COVID-19, Epistemic Injustice, Testimonial Injustice, Social disability model 


\section{Abstract}

In Long Covid, symptoms do not resolve within several weeks after acute infection with SARS-CoV-2. Patients with COVID-19 and long COVID face stigma and discrimination. One important type of discrimination is epistemic injustice which includes testimonial and hermeneutical injustices. Testimonial injustices occur when healthcare professional disregard or discount patients' symptoms. This worsens healthcare outcomes and exacerbates challenges to adequate healthcare access at the individual level. Furthermore, testimonial injustices may lead to hermeneutical injustices - systemic underrepresentation of the experiences of the marginalized and minoritized. Healthcare professionals play an essential role in mitigating injustices and have a duty to reduce harms done to patients with long COVID. It is crucial that the clinician avoid multiplying associated harms by not engaging in epistemic injustices.

We suggest the disability model as an approach to improve clinicians' response to long covid. Epistemic injustice leads to systemic inequities that affect those with chronic disease in general, particularly in populations suffering from structural inequities. The COVID-19 pandemic provides an opportunity for solidarity with those suffering from other chronic diseases that have been marginalized and stigmatized. 


\section{Introduction}

Since the beginning of the pandemic, COVID-19 has been associated with stigma and discrimination ${ }^{1}$. Such stigmatization is not limited to patients acutely ill with, and recovering from, COVID-19: survivors and healthcare workers have been denied services and suffered violent attacks throughout the world ${ }^{2,3}$. Emerging data suggests that between 1 in 10 to 1 in 3 COVID-19 survivors do not recover by 2-3 weeks after their primary infection ${ }^{4,5}$. Long COVID is a term originating with patients which highlights the duration and diversity of symptoms manifested by survivors, medical and socio-political challenges faced by patients, and a call to advocate and mitigate injustices ${ }^{6,7}$. The stigma associated with infectious diseases such as HIV/AIDS, SARS, Influenza, and acute COVID-19 ${ }^{1,8}$, additionally suggests that long COVID patients are at risk of healthcare discrimination.

Health-related stigma and discrimination are intersecting and mutually reinforcing phenomena ${ }^{9}$. In one example of discrimination driven by stigma ,patients with long COVID are not believed or their experiences are discounted by healthcare providers. This type of healthcare discrimination is known as epistemic injustice, which includes testimonial and hermeneutical injustice ${ }^{10,11}$.Testimonial injustice discounts individual experience based on prejudice; hermeneutical injustice involves systematic underrepresentation of the experiences of the marginalized and minoritized ${ }^{12}$. Testimonial injustice may lead to hermeneutical injustice ${ }^{12}$, meaning that if the testimony of long COVID patients are at high risk of not being recognized, then long COVID patients as a group are at high risk of epistemic injustice.

While more research is required to understand the prevalence of Long COVID among ethnic and racial minorities, ethnic and racial minorities have, in many countries, been more exposed to infection with SARS-CoV-2, and it is therefore possible that they will be disproportionately affected by Long COVID as well ${ }^{13}$. Ethnic and racial minorities encounter baseline epistemic injustice, disadvantage and vulnerabilities making them more susceptible to poor healthcare outcomes and decreased quality of care. ${ }^{14,15}$, Intersectional injustice (faced by those with identities with multiple minoritized or disadvantaged characteristics) should also be recognized in long COVID due to compound effects ${ }^{16}$. For 
example, a the symptoms presented by a Latina woman may be less believed than those of either a white woman or a white man. Moreover, stigma, which is "a process rooted in the ways that society negatively views individual or group characteristics or identities"17 can be reinforced through interaction with other systemic inequities, e.g. racism and poverty; thus increasing the complexity of health care ${ }^{18}$. Clinicians must ensure that epistemic injustice is not perpetrated.

\section{Long COVID and Testimonial Injustice}

"Patients' testimonies are often dismissed as irrelevant, confused, too emotional, unhelpful, or time-consuming" ${ }^{11}$. For example, women may be prescribed lower doses of narcotics when their pain is not validated but rather dismissed as anxiety ${ }^{19}$. Similarly, people of color and other minorities (e.g. Latinx) are likely to have their pain undertreated due to pain assessment and management disparities ${ }^{20}$.

Long COVID - as a new illness precipitated by infection with a new virus - poses particular risks of epistemic injustice. It includes symptoms that can be objectively measured by clinical examination and/or investigations (e.g. impaired heart function) and symptoms that entirely rely on the patient's testimony (e.g. fatigue) ${ }^{21}$. Symptoms that currently rely on the patient's testimony include both "subjective" symptoms such as pain, anxiety, and fatigue and other symptoms that are likely not detected through diagnostic workup. These symptoms are at higher risk of testimonial injustice since patients depend on their healthcare practitioner to acknowledge their condition and suffering in the absence of objective biomarkers. Furthermore, some patients who likely have had COVID-19 and now have long COVID might nevertheless have not been tested for the virus or have a negative test for the virus, and thus might be further dismissed on that basis, with their symptoms unacknowledged $^{22}$. If a condition is dismissed or misdiagnosed patients may be exposed to major health-related and other consequences. Take the following hypothetical case of Mr. L.C.:

Mr. L.C. is a 29 year old man who was healthy until diagnosis with probable COVID-19 two months ago. He presents at the clinic with fatigue and low energy since then. Mr. L.C. does 
not have a previous history of respiratory illness, cardiac illness, or fatigue. He did not require hospitalization; his predominant symptoms during COVID-19 acute phase were fever, cough, and fatigue. Physical exam is unremarkable and COVID testing is negative. Mr. L.C. is worried about his fatigue and energy level. His employer informed him that he is expected to return to work next week, but Mr. L.C. is worried that his fatigue and lack of energy will hinder his performance. Due to the pandemic and high unemployment rates, his is the only income in his household. Lastly, Mr. L.C. mentions that he is extremely worried about re-infection with COVID-19. He also says "I have been going to so many physicians, but nobody takes me seriously. They always dismiss me as if my symptoms were in my head. I don't know what else to do or where to go anymore."

If Mr. L.C.'s clinician believes that his fatigue is not related to COVID-19 then the clinician might be engaging in (or at best complicit in) epistemic injustice. Depending on Mr. L.C.'s sociodemographic background and ethnicity, the risk of epistemic injustice might worsen. For example, if Mr. L.C. is Black or Latinx, a woman with similar economic hardships, an immigrant, and so on. If the practice continues and more Long COVID patients are dismissed as suffering from "something else," such that the pool of collective knowledge regarding patients with persisting COVID illness is depleted of their accurate experiences, then patients with long COVID will also be facing hermeneutical injustice.

\section{Reducing Harm Done to Long COVID patients}

In the case of Long COVID, a harm reduction approach might mitigate the current harms and challenges encountered by Long COVID patients while seeking medical recognition and care for their health, rather than placing blame and/or responsibility on the patient to "prove" their illness. A harm reduction approach in this case would prioritize aiding the patient instead of assuming that the patient seeks to avoid work without reason, or that the patient is "guilty" of having COVID-19 in the first place due to their behavior or not seeking laboratory diagnosis early in the illness. Clinicians can help patients navigate the pandemic by making a clinical diagnosis of Long COVID, recognizing debilitating conditions, providing treatment and advice, locating resources available in the community, and advocating for measures to enable all those with COVID to live full lives in the context of their illness while preserving others' safety ${ }^{23,24}$. 
Unfortunately, COVID-19 infections will continue to occur and clinicians should recognize that at least $10 \%$ of the overall population with acute COVID-19 are likely to get Long COVID ${ }^{4,21}$. Patients from ethnic minorities and women seem to be more at risk 21, 25-27. Patients like Mr. L.C. should be evaluated holistically considering a full differential diagnosis, so that any underlying disease(s) or condition(s) can be responsibly treated (Table 1). If the clinician's practice enacts or is complicit in testimonial injustice, then the clinician will be multiplying associated harms. Clinicians need to acknowledge and address patients' symptoms and recognize the challenges of epistemic injustice ${ }^{15}$.

Table 1: Mitigating Epistemic Injustice in Long Covid: Fundamental Steps

\section{Clinical Evaluation}

Acknowledge, validate patients' health complains.

Reduce epistemic Injustice, bias, stigma, and discrimination

\section{Diagnosis or Differential Diagnosis Including Long COVID} Awareness that Long COVID is a multisystemic disease Recognize Long COVID as an entity of its own whose mechanism(s) are not currently understood.

\section{Clinical assessment including laboratory and other work-up to exclude underlying treatable pathology}

\section{Appropriate treatment and rehabilitation}

Supporting patients' request to apply to disability benefits when appropriate.

Misdiagnosis, failing to validate patients' experience or take it seriously, interpreting their experience very differently from the patient themselves-- all of these may contribute to anxiety 28. At the same time, the COVID-19 pandemic has reinforced risk factors for anxiety, such as health, economic, and social concerns ${ }^{29}$. In other words, due to the vicious cycle of the pandemic and anxiety, clinicians should be ready to expect high levels of anxiety, while not automatically identifying every potential symptom with anxiety. Currently we do not have a concrete or unifying description of Long COVID. For that reason, we consider it an urgent priority to progress beyond an anecdotal account of this phenomenon ${ }^{30}$. In addition, an effective 
response to the pandemic must consider sequelae beyond mortality and consider measuring prolonged ill health ${ }^{31}$. Therefore, it is crucial that healthcare professionals are aware of epistemic injustice, endeavoring to reduce harm to patients while validating, understanding, and - where appropriate -- assessing and documenting their experience.

\section{Social Disability Model}

Some long COVID-19 symptoms are debilitating and disabling; for example long term lung function impairment as well as cardiac and neurological sequelae ${ }^{21}$. Long COVID patients will require holistic and comprehensive post-acute care ${ }^{21}$. Besides coping with their new health conditions, long COVID patients with disabling symptoms will likely encounter social, economic, and physical inequities ${ }^{29}$. How can a clinician address this?

The social disability model proposes that disability inheres not in the individual, but in the attitudes and structures of society ${ }^{32}$. The social disability model demands societal adjustment to minimize barriers encountered by disabled people ${ }^{29}$. Healthcare professionals are a key component for helping patients adjust to their new life and avoid healthcare discrimination, and this model can help us further understand the care of Mr. L.C.

After a few weeks Mr. L.C. returns to the clinic. His fatigue has not improved even after performing the recommended exercises. He also informs you that his employer is displeased with his job performance; he is fearful of losing the only income in his household. He saw on the $C D C$ 's website that some people with chronic fatigue may qualify for disability benefits. He wants to apply for disability benefits and has brought the forms.

The above discussions of epistemic injustice and social disability theory help us understand how a clinician might structure their response. Clinicians should provide encouragement and hope to their patients -- even more so when an ongoing condition like Long COVID is not obviously or necessarily susceptible to curative treatments. It is the clinician's job to listen to and believe the patient, reassess symptoms to improve health quality, help the patient navigate often unresponsive systems (including supporting patients' request to apply to disability benefits when appropriate), and confront those systems (ideally changing them) with the reality of patient experience. 


\section{Solidarity with Those with Chronic Diseases}

Given the huge numbers of patients likely to be experiencing persistent COVID-19 symptoms, increasing awareness of long COVID is essential. It is also important to recognize that patient communities with chronic illness - such as myalgic encephalomyelitis/chronic fatigue syndrome (ME/CFS) - are neglected and also experience epistemic injustice ${ }^{33}$. Similar to Long COVID, $\mathrm{ME} / \mathrm{CSF}$ has no specific laboratory test or cure; thus, healthcare providers must rely in the evaluation of symptoms and medical history. In other words, ME/CSF patients are at a similar risk of epistemic injustice, and associated deleterious healthcare outcomes ${ }^{33}$.

However, we suggest that Long COVID is distinct in several aspects compared to other chronic conditions. First, Long COVID is a novel disease with a known pathogen. In this setting, survivors reporting symptoms beyond 4 weeks are doing so in an atmosphere of pre-existing uncertainty regarding the disease as a whole. Second is stigma and its intersectionality: while chronic pain, ME/CFS and other conditions (e.g. chronic Lyme) are certainly associated with significant stigma, discrimination, and marginalization, an infectious disease in the context of a current pandemic gives rise to a particularly virulent type of stigma; which can potentially be embodied based on social identities (e.g. disease-related stigma and race-related stigma). Third, COVID-19 is a threat to the survival and health of a population around the world, and long COVID is associated with that threat, unlike the other above mentioned chronic diseases. Lastly, COVID-19 is affecting more people in a much shorter span of time.

Despite certain differences from established chronic conditions, Long COVID can assist in understanding the struggles and mitigating the injustices faced by similar populations. It provides an opportunity for solidarity in facing stigma, marginalization, and epistemic injustice by researching and studying the struggles faced by the population (e.g. health discrimination) and responses (e.g. policies). As well as rectifying systemic inequities affecting all those with chronic diseases and particularly populations suffering from structural inequities.

\section{Conclusion}

Long COVID patients who do not return to their previous baseline condition are likely to struggle during adjustment to this new state and may encounter discrimination. Healthcare providers have an essential role which includes validation, guiding, and advocating for their patients' wellbeing. Individuals vulnerable to health and non-health related stigma and certain health conditions are at a higher risk of epistemic injustice, and thus increased risk of healthcare 
discrimination. Healthcare providers must adequately prepare for the long COVID patient surge by acknowledging and mitigating both testimonial and hermeneutical injustices, understanding how societal structures magnify the deleterious effects of chronic disease.

\section{Statements}

All authors have contributed to the drafts and final version of the manuscript. There is no conflict of interest, no funding, and ethics committee approval does not apply for this article. 


\section{References}

1. Logie $\mathrm{CH}$, Turan JM. How do we balance tensions between COVID-19 public health responses and stigma mitigation? Learning from HIV research. AIDS and Behavior. 2020:1-4. 2. Bagcchi S. Stigma during the COVID-19 pandemic. The LancetInfectious Diseases. 2020;20(7):782.

3. Singh R, Subedi M. COVID-19 and stigma: Social discrimination towards frontline healthcare providers and COVID-19 recovered patients in Nepal. Asian journal of psychiatry. 2020;

4. Tenforde MW, Kim SS, Lindsell CJ, et al. Symptom duration and risk factors for delayed return to usual health among outpatients with COVID-19 in a multistate health care systems network-United States, March-June 2020. Morbidity and Mortality Weekly Report. 2020;69(30):993.

5. Sudre $\mathrm{CH}$, Murray B, Varsavsky T, et al. Attributes and predictors of Long-COVID: analysis of COVID cases and their symptoms collected by the Covid Symptoms Study App. medRxiv. 2020;

6. Perego E, Callard F, Stras L, Melville-Jóhannesson B, Pope R, Alwan NA. Why the Patient-Made Term'Long Covid'is needed. Wellcome Open Research. 2020;5(224):224.

7. Callard F, Perego DE. How and Why Patients Made Long Covid. Social science \& medicine. 2020:113426. doi:https://doi.org/10.1016/i.socscimed.2020.113426

8. Williams J, Gonzalez-Medina D. Infectious diseases and social stigma. Applied Innovations and Technologies. 2011;4(1):58-70.

9. Stangl AL, Earnshaw VA, Logie $\mathrm{CH}$, et al. The health stigma and discrimination framework: a global, crosscutting framework to inform research, intervention development, and policy on health-related stigmas. BMC medicine. 2019;17(1):1-13.

10. Fricker M. Epistemic injustice: Power and the ethics of knowing. Oxford University Press; 2007.

11. Carel H, Kidd IJ. Epistemic injustice in healthcare: a philosophial analysis. Medicine, Health Care and Philosophy. 2014;17(4):529-540.

12. Byskov MF. What Makes Epistemic Injustice an "Injustice"? Journal of Social Philosophy. 2020;

13. Runnymede. Covid-19's Impact on BME Communities. 2020.

14. Tai DBG, Shah A, Doubeni CA, Sia IG, Wieland ML. The disproportionate impact of COVID-19 on racial and ethnic minorities in the United States. Clinical Infectious Diseases. 2020;

15. McConkey J. Knowledge and acknowledgement:'epistemic injustice'as a problem of recognition. Politics. 2004;24(3):198-205.

16. Kelly UA. Integrating intersectionality and biomedicine in health disparities research. Advances in Nursing Science. 2009;32(2):E42-E56.

17. Turan JM, Elafros MA, Logie $\mathrm{CH}$, et al. Challenges and opportunities in examining and addressing intersectional stigma and health. BMC medicine. 2019;17(1):1-15.

18. Logie $\mathrm{CH}$. Lessons learned from HIV can inform our approach to COVID-19 stigma. Journal of the International AIDS Society. 2020;23(5):e25504.

19. Hoffmann DE, Tarzian AJ. The girl who cried pain: a bias against women in the treatment of pain. The Journal of Law, Medicine \& Ethics. 2001;28:13-27.

20. Johnson-Jennings M, Duran B, Hakes J, Paffrath A, Little MM. The influence of undertreated chronic pain in a national survey: Prescription medication misuse among American indians, Asian Pacific Islanders, Blacks, Hispanics and whites. SSM-Population Health. 2020:100563. 
21. Greenhalgh T, Knight M, Buxton M, Husain L. Management of post-acute covid-19 in primary care. bmj. 2020;370

22. Alwan NA, Attree E, Blair JM, et al. From doctors as patients: a manifesto for tackling persisting symptoms of covid-19. bmj. 2020;370

23. Kingstone T, Taylor AK, Donnell CA, Atherton H, Blane DN, Chew-Graham CA. Finding the 'right' GP: a qualitative study of the experiences of people with long-COVID. BJGP Open. 2020:bjgpopen20X101143. doi:10.3399/bjgpopen20X101143

24. Ladds E, Rushforth A, Wieringa S, et al. Persistent symptoms after Covid-19: qualitative study of 114 "long Covid" patients and draft quality criteria for services. medRxiv.

2020:2020.10.13.20211854. doi:10.1101/2020.10.13.20211854

25. Mahajan UV, Larkins-Pettigrew M. Racial demographics and COVID-19 confirmed cases and deaths: a correlational analysis of 2886 US counties. Journal of Public Health. 2020;

26. Moore JT, Ricaldi JN, Rose CE, et al. Disparities in Incidence of COVID-19 Among Underrepresented Racial/Ethnic Groups in Counties Identified as Hotspots During June 5-18, 2020 -- 22 States, February-June 2020. MMWR: Morbidity \& Mortality Weekly Report. 2020;69(33):1122-1126.

27. Kidd IJ, Carel H. Epistemic injustice and illness. Journal of applied philosophy. 2017;34(2):172-190.

28. Asmundson GJG, Taylor S. How health anxiety influences responses to viral outbreaks like COVID-19: What all decision-makers, health authorities, and health care professionals need to know. Journal of anxiety disorders. 2020;71:102211.

29. Burchardt* T. Capabilities and disability: the capabilities framework and the social model of disability. Disability \& society. 2004;19(7):735-751.

30. Alwan NA. A negative COVID-19 test does not mean recovery. Nature. 2020;584(7820):170-170. doi:10.1038/d41586-020-02335-z

31. Alwan NA. Surveillance is underestimating the burden of the COVID-19 pandemic. The Lancet. 2020;396(10252):e24.

32. Haegele JA, Hodge S. Disability Discourse: Overview and Critiques of the Medical and Social Models. Quest (00336297). 2016;68(2):193-206.

33. Blease C, Carel H, Geraghty K. Epistemic injustice in healthcare encounters: evidence from chronic fatigue syndrome. Journal of medical ethics. 2017;43(8):549-557. 